\title{
Comparação de critérios de seleção para precocidade de crescimento em bovinos da raça Guzerá ${ }^{1}$
}

\author{
Aila Loise Ribeiro Tanaka ${ }^{2,3}$, Roberto Carvalheiro ${ }^{4,5}$, Luiz Alberto Fries $^{2,5^{*}}$, Sandra Aidar de Queiroz ${ }^{6}$ \\ 1 Projeto financiado pelo CNPq. \\ 2 CRV Lagoa - Sertãozinho - SP - Brasil. \\ 3 Programa de Pós-Graduação em Genética e Melhoramento Animal - FCAV - Unesp. \\ ${ }^{4}$ Bolsista FAPESP. \\ ${ }^{5}$ GenSys Consultores e Associados S/C Ltda - Porto Alegre - RS - Brasil. \\ ${ }^{6}$ Departamento de Zootecnia - FCAV - Unesp - Jaboticabal - SP - Brasil - Bolsista CNPq. \\ *In memoriam.
}

RESUMO - Objetivou-se com este estudo comparar a seleção com base no ganho médio diário de peso na pré-desmama (GMD) e no número de dias para ganhar 160 kg nessa fase (D160), com e sem correção para efeitos de grupos de contemporâneos (GC), em bovinos da raça Guzerá. Utilizou-se o banco de dados de desenvolvimento ponderal da Associação Brasileira de Criadores de Zebu (ABCZ) para esta raça. A obtenção dos parâmetros e valores genéticos foi feita pelo método da máxima verossimilhança restrita utilizando-se modelo unicaracterístico com equações de modelos mistos. O modelo utilizado foi composto pelo efeito fixo de grupo genético e pelos efeitos aleatórios, genético aditivo direto e de ambiente permanente, além do erro residual. A média para D160 foi de 270,5 dias e para GMD, 642,3 g. As correlações de Spearman entre ganho médio diário e a precocidade em dias para ganhar 160 kg na pré-desmama (PD160), e GMD e PD160c (PD160 corrigido para o efeito de grupo de contemporâneo), foram iguais a 0,91 e 0,94, respectivamente. A seleção para PD160 favorece touros que produzem progênie com desempenho superior e menos variável e a padronização deste critério com base no grupo de contemporâneos melhorou sua eficiência. A classificação dos touros varia de acordo com o critério de seleção utilizado, GMD ou PD160, principalmente nos extremos, onde ocorrem seleção e descarte de reprodutores.

Palavras-chave: dias para ganhar 160 kg na pré-desmama, ganho médio diário, média harmônica, progênie uniforme

\section{Comparison of selection criteria for growth precocity in Guzerat cattle}

ABSTRACT - The aim of this study was to compare the selection criteria based on the average daily gain (ADG) and days to achieve $160 \mathrm{~kg}$ (D160) on pre-weaning of Guzerat cattle. Database on weight gains provided by the Brazilian Association of Zebu Cattle Breeders (ABCZ) was used. The estimated genetic parameters and breeding values was obtained by the Restricted Maximum Likelihood Method, using a uni-trait animal model, with equations of mixed models. The model included the fixed effect of contemporary group, genetic additive direct and permanent environment, as random effects, beyond the residual error. The means for D160 and ADG were 270.5 days and 642.3 g, respectively. Spearman's correlations for pre-weaning ADG and D160 (D160P), and ADG and D160P corrected for contemporary group effect (D160Pc), were equal to 0.92 and 0.94 , respectively. The selection for D160P would favor sires that produced superior and less variable progenies. The correction of D160 to the contemporary group effect made this trait more efficient to identify more uniform sires. Sires classification changed according to the adopted selection criterion, ADG or D160P, mainly at the end points, where animals should be selected and rejected.

Key Words: average daily gain, days to achieve $160 \mathrm{~kg}$ on pre-weaning, harmonic mean, uniform offspring

\section{Introdução}

O melhoramento genético de bovinos de corte para precocidade de crescimento no período pré-desmama tem sido baseado em critérios de seleção que incluem o peso em determinada idade ou peso à desmama ajustado para 205 dias, sempre almejando animais com altos valores para peso.
Essa seleção origina respostas correlacionadas, como aumento no peso em idades subseqüentes, favorável nos machos. Entretanto, se aplicada indevidamente, o incremento no tamanho da vaca, na maioria das vezes, não é desejado, o que ocasiona desequilíbrio entre as exigências de crescimento, reprodução e manutenção do animal adulto, inviáveis em sistemas de produção em pastagens (Fries et al., 1996). 
O ganho médio diário do nascimento à desmama (GMDND) é a relação entre duas diferenças: o numerador é a diferença entre os pesos medidos à desmama e ao nascimento, enquanto que o denominador é a diferença entre as datas em que estes eventos ocorreram (Fries \& Albuquerque, 1996). Embora esta medida também sofra efeitos maternos, não é influenciada pelo peso ao nascer e reflete um pouco melhor o desempenho do indivíduo na pré-desmama. Assim, a utilização do GMDND como critério de seleção poderia diminuir o impacto no aumento do peso ao nascer, como resposta indireta à seleção aplicada sobre ganho médio diário do nascimento à desmama.

Ortiz Peña et al. (2004) e Muniz et al. (2005a) sugeriram como alternativa o emprego de critérios de seleção que resultem em animais mais eficientes em ganho de peso em determinado tempo, como número de dias para o animal ganhar $160 \mathrm{~kg}$ entre o nascimento e a desmama (D160). Essa abordagem da precocidade de crescimento foi inicialmente proposta por Fries et al. (1996), que afirmaram que a ênfase seletiva em critérios de seleção utilizando o numerador do ganho médio diário e os pesos corrigidos pode ser interpretada como a busca por um animal maior. A comparação entre a seleção com base nos "dias necessários para atingir determinado peso” e “ganho médio diário de peso” tem sido relatada na literatura (Roso, 1997; Pita \& Albuquerque, 2001).

Outro aspecto relevante é que as avaliações genéticas usuais, baseadas em características como pesos ajustados a determinadas idades ou GMDND, geram estimativas pontuais dos parâmetros do modelo, que, por sua vez, são "funções” da média aritmética dos valores observados destas características. A característica D160, por outro lado, apresenta propriedades inerentes à média harmônica, o que favorece touros cuja progênie tem desempenho menos variável (Ortiz Pena et al., 2004).

Este trabalho foi realizado para avaliar a seleção para precocidade de crescimento em bovinos da raça Guzerá, realizada com base no GMDND, D160 e D160, obtido a partir da correção do GMDND para o efeito de grupo de contemporâneos (D160c), e comparar (ou discriminar) estes critérios de seleção pelos valores genéticos preditos.

\section{Material e Métodos}

Utilizaram-se dados do Controle de Desenvolvimento Ponderal de bovinos da raça Guzerá, pertencentes ao banco de dados zootécnicos da Associação Brasileira dos Criadores de Zebu (ABCZ). A formação e consistência do arquivo de dados foram realizadas utilizando-se o programa computacional SAS $^{\circledR}$ (SAS, 1996).
Na estimação do ganho médio diário de peso no período pré-desmama (GMDND), recorreu-se a procedimentos de ajustes usuais pré-avaliação genética, como: correção para 205 dias, conforme a idade do bezerro no período; ajustado para a idade da vaca ao parto (IVP), conforme o sexo do bezerro e ajustado para a data juliana (DJN) média dentro de cada estação de nascimento.

O cálculo da variável D160 foi feito da seguinte forma: D160 = 160/GMDND, em que: D160 = número de dias necessários para o animal atingir 160 kg no período pré-desmama; 160 = ganho de peso $(\mathrm{kg})$ correspondente a um produto desmamado com peso em torno de $190 \mathrm{~kg}$; GMDND = ganho médio diário do nascimento à desmama (kg/dia), corrigido para 205 dias de idade, idade da vaca ao parto de acordo com o sexo do bezerro e a data juliana.

O grupo de contemporâneos (GC) foi formado pela concatenação dos efeitos de criador, fazenda do criador, sexo, ano de nascimento, estação de nascimento, registro (puro de origem ou livro aberto) e regime alimentar, correspondendo a 1.814 GC, contemplando filhos de 611 touros e de 14.789 vacas, total de 26.633 animais, de uma matriz de parentesco de 39.712 animais.

$\mathrm{O}$ arquivo de dados foi analisado com o programa computacional MTDFREML (Boldman et al., 1993) e as estimativas dos componentes de variância e herdabilidade foram obtidas pelo método da Máxima Verossimilhança Restrita, por meio de modelo animal unicaracterístico com equações de modelos mistos. O modelo empregado nas análises foi: $\mathrm{y}=\mathrm{Xb}+\mathrm{Za}+\mathrm{Wp}+\mathrm{e}$, em que: $y=$ vetor das observações pré-ajustadas para o GMDND e D160; X = matriz da incidência associada aos efeitos fixos; $b=$ vetor dos efeitos de grupo de contemporâneos (fixos); Z, W = matrizes de incidência associadas aos efeitos aleatórios; $\mathrm{a}=$ vetor do efeito genético aditivo direto (AD) do animal (aleatório); $\mathrm{p}$ = vetor do efeito de ambiente permanente (AP), (aleatório); e = vetor dos resíduos aleatórios, não observáveis.

No modelo considerado para avaliação genética, constaram as seguintes pressuposições:

$\mathrm{E}=\left[\begin{array}{c}\mathrm{y} \\ \mathrm{a} \\ \mathrm{p} \\ \mathrm{e}\end{array}\right]=\left[\begin{array}{c}\mathrm{Xb} \\ 0 \\ 0 \\ 0\end{array}\right] \quad \mathrm{VAR}=\left[\begin{array}{c}\mathrm{a} \\ \mathrm{p} \\ \mathrm{e}\end{array}\right]=\left[\begin{array}{ccc}\sigma_{\mathrm{a}}^{2} \mathrm{~A} & 0 & 0 \\ 0 & \sigma_{\mathrm{p}}^{2} \mathrm{I}_{\mathrm{C}} & 0 \\ 0 & 0 & \sigma_{\mathrm{e}}^{2} \mathrm{I}_{\mathrm{N}}\end{array}\right]$

em que: $\mathrm{A}=$ numerador da matriz de parentesco; $\mathrm{N}$ = número de registros; $\mathrm{I}=$ matriz identidade; $\mathrm{C}$ = número de vacas; $\sigma_{a}^{2}=$ variância genética aditiva direta; $\sigma_{p}^{2}=$ variância de ambiente permanente; $\sigma_{e}^{2}=$ variância residual.

As soluções para os parâmetros de interesse do modelo foram obtidas segundo as Equações de Modelos Mistos de 
Henderson (Henderson, 1984) e podem ser descritas matricialmente por:

$$
\left[\begin{array}{ccc}
X^{\prime} X & X^{\prime} Z & X^{\prime} W \\
Z^{\prime} X & Z^{\prime} Z+A^{-1} k_{1} & Z^{\prime} W \\
W^{\prime} X & W^{\prime} Z & W^{\prime} W+I k_{2}
\end{array}\right]\left[\begin{array}{c}
\hat{b} \\
\hat{a} \\
\hat{p}
\end{array}\right]=\left[\begin{array}{c}
X^{\prime} y \\
Z^{\prime} y \\
W^{\prime} y
\end{array}\right],
$$

em que: $\mathrm{k}_{1}=\sigma_{e}^{2} / \sigma_{a}^{2}, \mathrm{k}_{2}=\sigma_{e}^{2} / \sigma_{p}^{2}$, e A $\mathrm{A}^{-1}$ a inversa da matriz de parentesco.

O efeito aditivo materno não foi incluído em virtude do confundimento na sua estimação quando o efeito de AP foi incluído no modelo, provavelmente em decorrência do número pequeno de filhos por vaca (1,8 filhos/vaca), o que não permitiu separar e estimar de forma adequada os componentes de efeito materno. Com a inclusão dos dois componentes de efeito materno, apenas um deles era estimado, enquanto o outro era igualado a zero. Portanto, a decomposição do componente materno não melhorou o ajuste para esse conjunto de dados.

Utilizaram-se as correlações entre os valores genéticos dos animais, obtidos com base nas características consideradas, como alternativa de comparação dos critérios de seleção estudados. Para isso, realizaram-se análise de correlação de Spearman do procedimento CORR do programa computacional SAS ${ }^{\circledR}$ (SAS, 1996) e correlação Pearson, estimada pelo mesmo procedimento.

Para facilitar a interpretação dos resultados, os valores genéticos estimados para D160 foram multiplicados por (-1) para obter dias a mais em precocidade (PD160), recomendado por Muniz et al. (2005b). Assim, valores genéticos positivos para PD160 são desejáveis.

Outro procedimento utilizado foi classificar os indivíduos segundo seus valores genéticos preditos para GMDND e PD160 e categorizá-los em lotes de classes percentuais de 10 em 10\%, denominados decas. As decas foram calculadas considerando os 611 touros avaliados. Posteriormente, avaliaram-se as mudanças de posição entre as decas, ou seja, animais que foram classificados em determinada deca para uma característica e, ao serem classificados pelo outro critério de seleção, mudaram de deca.

Outro método de comparação dos critérios de seleção foi o estudo da relação entre a média aritmética (A), a média harmônica $(H)$ e o desvio-padrão $(\sigma)$ das características GMDND e PD160. Para tanto, o GMDND foi corrigido para o desempenho de um mesmo grupo de contemporâneos e progênies de mesma vaca utilizando-se a equação:

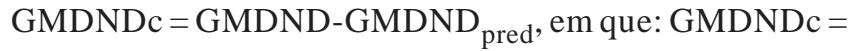
GMDND corrigido para o desempenho de mesmo grupo de contemporâneos; GMDND = ganho médio diário do nascimento à desmama; $\mathrm{GMDND}_{\text {pred }}=\mathrm{GMDND}$ predito obtido pela equação: $\mathrm{GMDND}_{\text {pred }}=\mathrm{sol}_{\mathrm{GC}}+(-\mathrm{md}) * \mathrm{k}+\mathrm{ap}$

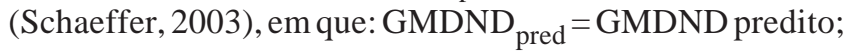
sol $_{\mathrm{GC}}=$ solução para o efeito de grupo de contemporâneos; md = solução para o efeito genético aditivo direto da vaca; $\mathrm{k}$ = variância residual/variância genética aditiva; ap = solução para o efeito de ambiente permanente.

Como alternativa para contornar problemas de heterogeneidade de variâncias entre grupos de contemporâneos apresentada por D160, recorreu-se a uma característica alternativa, D160c, segundo recomendações de Carvalheiro et al. (2003). A característica D160c foi obtida pela equação: $\mathrm{D} 160 \mathrm{c}=160 / \mathrm{GMDNDc}$, em que: $\mathrm{D} 160 \mathrm{c}=$ D160 corrigido para o efeito de grupo de contemporâneos; 160 = ganho de peso (em kg) correspondente a um produto desmamado com peso em torno de $190 \mathrm{~kg}$; GMDNDc = GMDND corrigido para o efeito de grupo de contemporâneos (kg/dia).

Foram então obtidas as médias aritmética (A) e harmônica $(\mathrm{H})$, o desvio $(\sigma)$ e a diferença entre as médias aritmética e harmônica (A-H) do GMDNDc da progênie de cada touro. Foram mantidos nas análises touros com progênie superior a dez filhos e desempenhos de GMDNDc positivos.

A relação entre A, H e $\sigma$ do GMDNDc foi analisada utilizando-se o procedimento GLM do programa SAS ${ }^{\circledR}$ (SAS, 1996), com modelo restrito (sem intercepto), conforme descrito por Carvalheiro et al. (2003):

$$
\mathrm{Y}_{\mathrm{ij}}=\mathrm{b}_{1}\left(\mathrm{x}_{\mathrm{i}}\right)+\mathrm{b}_{2}\left(\mathrm{x}_{\mathrm{i}}\right)^{2}+\mathrm{e}_{\mathrm{ij}} \text {, }
$$

em que: $\mathrm{Y}_{\mathrm{ij}}=$ diferença entre média aritmética e média harmônica obtida a partir da definição da variável GMDNDc, referente ao j-ésimo touro; $b_{1}$ e $b_{2}=$ coeficientes de regressão linear e quadrático, respectivamente; $\mathrm{x}_{\mathrm{i}}=$ desvio-padrão do GMDNDc da progênie do j-ésimo touro, em g/dia; $\mathrm{e}_{\mathrm{ij}}=$ erro aleatório associado a cada observação ij, suposto normalmente distribuído com média zero e variância $\sigma^{2}$.

Para verificar a relação entre a distribuição dos valores de A-H, conforme o $\sigma$, e a classificação dos touros de acordo com os valores genéticos estimados para GMDND, PD160 e PD160c, esta última obtida pela multiplicação de D160c por (-1), foi estabelecida a comparação quando a classificação dos touros foi alterada em uma ou mais decas, em decorrência da mudança dos critérios de seleção.

\section{Resultados e Discussão}

A comparação da seleção com base no ganho médio diário de peso do nascimento à desmama (GMDND) em relação ao uso de precocidade em dias para ganhar $160 \mathrm{~kg}$ na pré-desmama (PD160) é feita a princípio por meio das 
Tabela 1 - Correlações de Pearson e de Spearman entre os valores genéticos diretos preditos para GMDND e PD160; e para o GMDND e PD160c de animais da raça Guzerá

\begin{tabular}{lcc}
\hline Característica & $\begin{array}{c}\text { Correlação de } \\
\text { Pearson }\end{array}$ & $\begin{array}{c}\text { Correlação de } \\
\text { Spearman }\end{array}$ \\
\hline GMDND e PD160 & $0,8671^{* * *}$ & $0,9194^{* * *}$ \\
GMDND e PD160c & $0,9415^{* * *}$ & $0,9439 * * *$ \\
\hline
\end{tabular}

GMDND = ganho médio diário na pré-desmama; $\mathrm{PD} 160$ = precocidade em dias para ganhar 160 kg; PD160c = PD160 corrigido para o efeito de grupo de contemporâneos. $\mathrm{P}<0,001$.

correlações de Pearson (amostral) e de Spearman (classificação) entre os valores genéticos diretos, preditos para o GMDND e PD160 e para o GMDND e PD160c (Tabela 1).

As correlações de Pearson estimadas foram positivas e de alta magnitude $(\mathrm{P}<0,001)$, indicando que parte dos genes que determinam a característica GMDND também afetam simultaneamente PD160 ou PD160c. Assim, quanto maior o GMDND, mais precoce o animal, ou seja, menor o tempo que levará para atingir a unidade de mercado estipulada.

A correlação entre GMDND e PD160 estimada neste trabalho foi semelhante à relatada por Muniz et al. (2005b) para animais da raça Gir. Entretanto, Fries et al. (1996) e Ortiz Peña et al. (2001) encontraram correlações maiores, 0,94 e 0,95 , respectivamente, entre GMDND e D160 para animais da raça Nelore. Essas diferenças nos valores de correlação entre as raças podem ser atribuídas ao equívoco de se estimar a eficiência de produção utilizando uma unidade de mercado não adequada para raça. O histórico de seleção aplicada às raças Gir e Guzerá indica que o critério D160 poderia ser adaptado. Muniz et al. (2005a) sugerem a necessidade de se definir o peso e número de dias para atingir esse peso no período pré-desmama específicos para a raça Gir.

As correlações de Spearman estimadas para verificar o grau de associação entre as posições de classificação dos touros para GMDND e PD160; e GMDND e PD160c foram, assim como a de Pearson, de alta magnitude e significativas (Tabela 1), maiores que as encontradas por Muniz et al. (2005b) para animais da raça Gir (0,90). Esse grau de associação indica que a maior parte dos touros teria a mesma classificação quando escolhidos por um ou outro critério de seleção, no entanto, baseando-se apenas na correlação, não seria possível detectar onde ocorre essa alteração, o que é importante em termos de seleção.

Este fato fica mais evidente quando se analisa a distribuição dos valores genéticos diretos das características PD160 e PD160c (Figuras 1 e 2) em relação aos valores genéticos diretos do GMDND, que indicam alteração na classificação dos touros com base nos valores genéticos diretos, como conseqüência da adoção de um ou outro critério de seleção, principalmente nos extremos, onde se situam os animais com melhor ou pior classificação. Muniz et al. (2005b) também observaram comportamento semelhante para a distribuição dos valores genéticos diretos de D160 em relação ao GMDND. Com o banco de dados da raça Gir, relataram dispersão nos valores extremos e concentração de pontos para valores genéticos próximos ou iguais a zero.

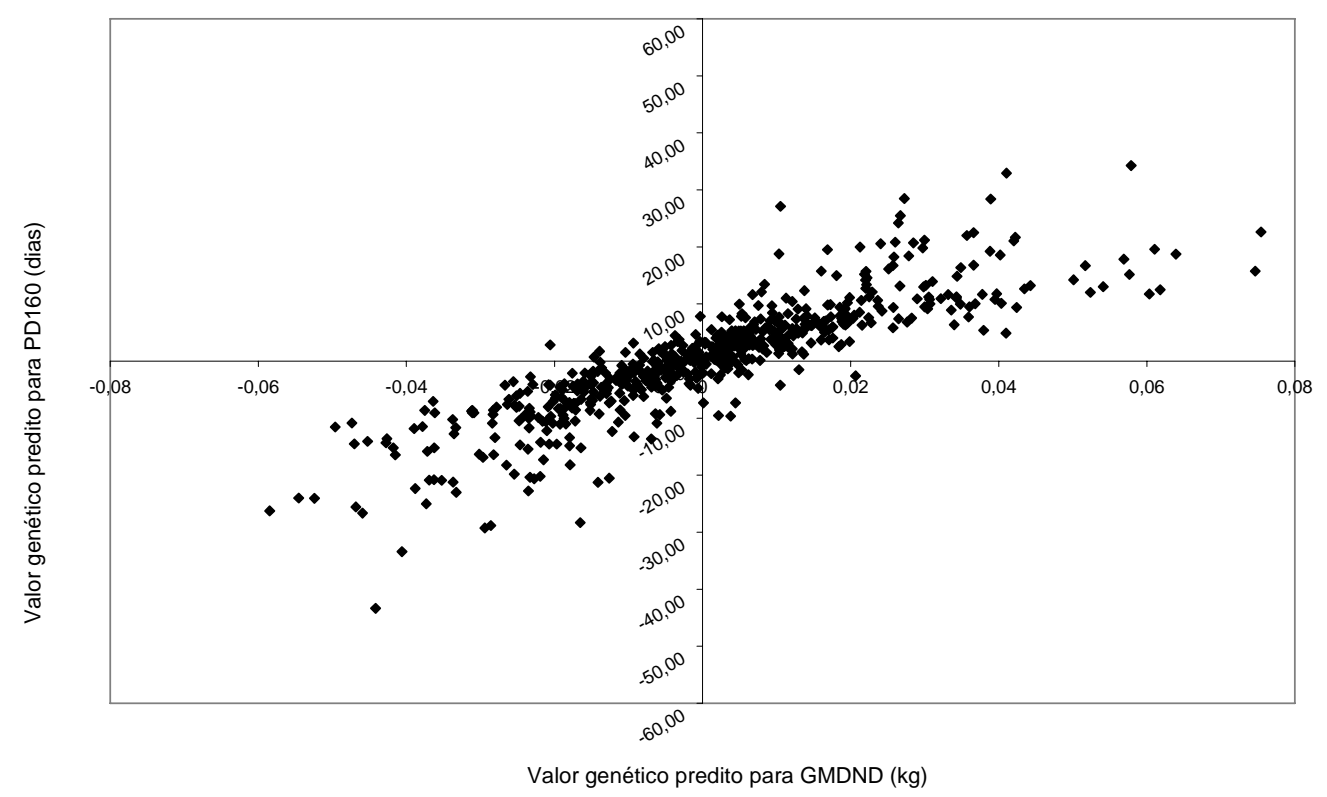

Figura 1 - Dispersão dos valores genéticos de efeitos diretos das características ganho médio diário na pré-desmama (GMDND) e precocidade em dias para ganhar 160 kg (PD160) em touros Guzerá 


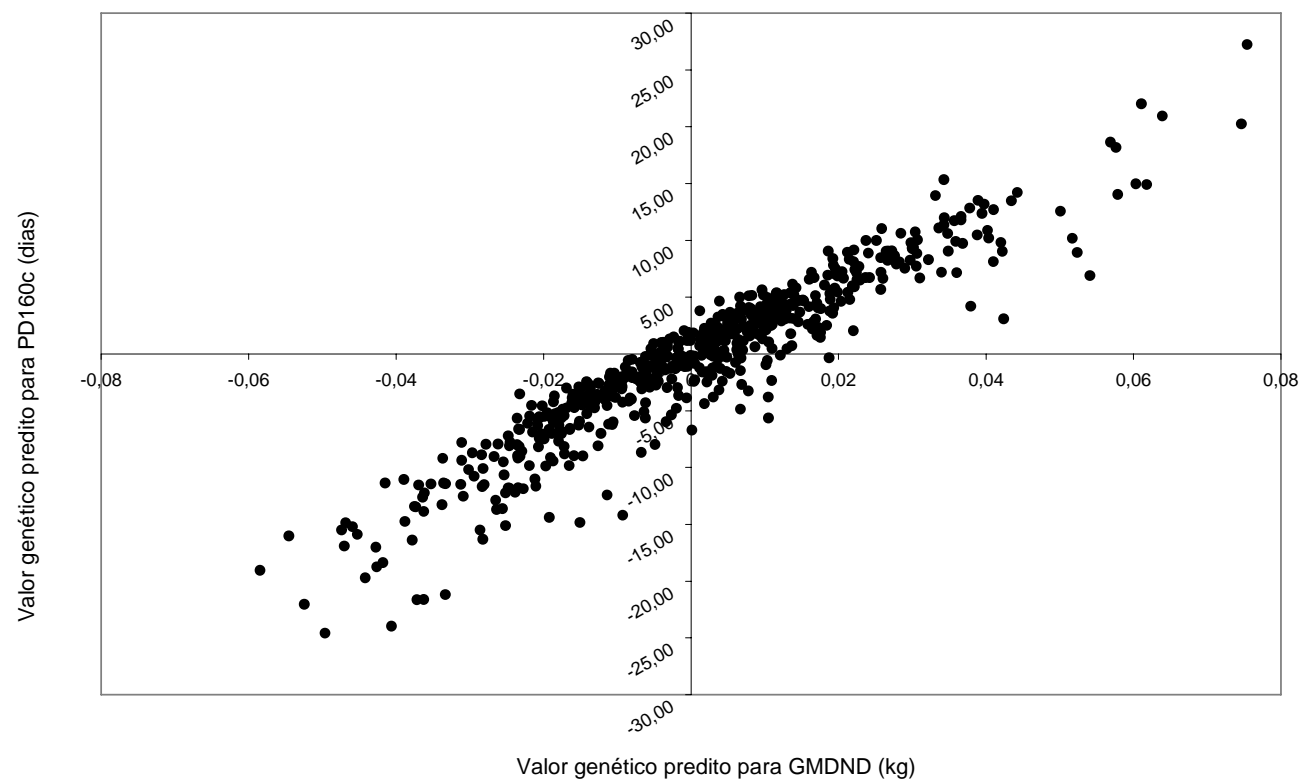

Figura 2 - Dispersão dos valores genéticos de efeitos diretos das características ganho médio diário na pré-desmama (GMDND) e precocidade em dias para ganhar 160 kg corrigidos para o efeito de grupo de contemporâneos (PD160c) em touros Guzerá.

As diferenças de decas negativas (Tabela 2) indicam que os touros foram melhor classificados quando usado GMDND, em comparação a PD160 ou PD160c, enquanto diferenças positivas indicam o oposto.

Numericamente, 239 touros não mudaram de classificação quando selecionados pelo GMDND ou pelo PD160, assim como 312 touros também não mudaram de decas quando a seleção foi feita com base no PD160c ou no GMDND. Esses valores representam 39,12 e 51,06\% dos reprodutores avaliados que não mudaram de deca quando foi alterado o critério de seleção, ou seja, de GMDND para PD160 ou para PD160c, respectivamente. Logo a correção pelo grupo de contemporâneos implicou diferença em torno de $10 \%$. O resultado observado para a seleção pelo GMDND ou D160 foi similar ao que constataram Muniz et al. (2005b) com a raça Gir.

A classificação de $21,60 \%$ dos touros foi melhor pelo GMDND, enquanto em 22,60\% dos touros foi melhor quando baseada no PD160. Esses valores representam a alteração de uma deca para outra. Valores similares foram observados por Muniz et al. (2005b) para a raça Gir e essa mudança de classificação ocorreu em 22,5\% dos touros dessa raça para D160 e em 19,7\% para GMDND.

Em comparação a GMDND, PD160c foi o melhor critério de seleção, pois possibilitou obter maior porcentagem (25,37\%) de touros com melhor classificação em uma deca para PD160c, enquanto apenas 11,95\% foram melhores classificados para GMDND.

Reprodutores classificados para decas 1 a 4 sofreram modificação de decas, em decorrência da mudança no
Tabela 2 - Freqüência de diferenças de classificação entre GMDND e PD160; e GMDND e PD160c de 611 touros da raça Guzerá

\begin{tabular}{lcc}
\hline $\begin{array}{l}\text { Diferença de } \\
\text { classificação } \\
\text { em decas }\end{array}$ & C_GMDND_C_PD160 & C_GMDND_C_PD160c \\
\cline { 2 - 3 } & \multicolumn{3}{c}{ Freqüência (\%) } \\
\hline-6 & $2(0,33)$ & $1(0,16)$ \\
-5 & $4(0,65)$ & $1(0,16)$ \\
-4 & $7(1,15)$ & $4(0,65)$ \\
-3 & $38(6,22)$ & $10(1,64)$ \\
-2 & $132(21,60)$ & $34(5,56)$ \\
-1 & $239(39,12)$ & $73(11,95)$ \\
0 & $136(22,26)$ & $312(51,06)$ \\
1 & $44(7,20)$ & $155(25,37)$ \\
2 & $6(0,98)$ & $1(0,27)$ \\
3 & $2(0,33)$ & 0 \\
4 & $1(0,16)$ & 0 \\
5 & 0 & $0,16)$ \\
\hline
\end{tabular}

GMDND = ganho médio diário na pré-desmama; PD160 = precocidade em dias para ganhar $160 \mathrm{~kg}$; PD160c = PD160 corrigido para o efeito de grupo de contemporâneos.

C_GMDND = classificação para GMDND; C_PD160 = classificação para PD160 e C_PD160c = classificação para PD160c.

critério de seleção (Tabelas 3 e 4). Considerando a porcentagem de não coincidência entre as classificações do GMDND e PD160 dos animais, evidencia-se que, tanto para os extratos de escolha dos reprodutores (deca 1) quanto para o de descarte (deca 10), aproximadamente $60 \%$ obtiveram classificação coincidente, independentemente do critério de seleção utilizado. Assim, os resultados de classificação não coincidentes indicam que $40 \%$ dos animais que seriam escolhidos por um critério de seleção não o seriam por outro, denotando o impacto que poderia ter a correção 
prévia para a fonte de variação na busca de precocidade de crescimento por meio da seleção baseada no PD160c.

Os coeficientes de regressão linear e quadrático e desvios-padrão para GMDNDc da progênie dos touros foram-0,0182 $\pm 0,0115$ e 1,8214 $\pm 0,0761$, com coeficiente de ajuste do modelo à variabilidade dos dados $\left(\mathrm{r}^{2}\right)$ igual a 0,9448 (Tabela 5).

Somente o efeito quadrático foi significativo $(\mathrm{P}<0,001)$, portanto, o desvio-padrão afetou a diferença entre as médias aritmética e harmônica. Desta forma, quanto maior o desvio-padrão, mais distante as medidas aritméticas ficaram das médias harmônicas (Figura 3). Assim, touros cujas progênies apresentaram, em média, os mesmos GMDNDc, mas com variabilidade diferente, não foram detectados pela média aritmética. O uso da média harmônica, no entanto, favoreceu touros com progênies mais uniformes.

As diferenças nos valores genéticos estimados das características GMDND e PD160 evidenciaram-se na variação da posição de classificação de -300 a 264 para touros melhores classificados para GMDND. Os valores extremos para a diferença do GMDND e PD160c (touros mais bem classificados para PD160c) foram -309 e 141 posições. Essa variação na classificação dos reprodutores foi maior que as encontradas por Carvalheiro et. al. (2003) para animais Nelore. Quando foram comparados os touros que apresentaram alteração na classificação em mais de 61 posições, ou seja, $10 \%$ do total de 611 touros avaliados para GMDNDc, 30,93\% foram melhores para PD160, enquanto 28,80\% foram melhores para PD160c, reafirmando a variação apresentada na seleção dos touros por um ou outro critério.

As propriedades da média harmônica foram mantidas em PD160 e PD160c, favorecendo touros que produziram progênie superior e de desempenho menos variável (Figuras 4 e 5). A proporção de touros classificados para GMDNDc foi maior que para PD160 e PD160c, quando estes apresentaram progênie com variabilidade acima do desviopadrão médio. Quando a progênie dos touros apresentou variabilidade menor que o desvio-padrão médio, a proporção de touros classificados para PD160 e Pd160c foi maior que a dos classificados para GMDNDc. Contudo, PD160c favoreceu touros cuja progênie apresentou menor variabilidade que PD160.

Tabela 3 - Mudança de classificação em decas com o uso dos critérios de seleção GMDND e PD160 para 611 touros da raça Guzerá

\begin{tabular}{|c|c|c|c|c|c|c|c|c|c|c|c|}
\hline \multirow{2}{*}{ Seleção } & \multicolumn{11}{|c|}{ GMDND } \\
\hline & Decas & 1 & 2 & 3 & 4 & 5 & 6 & 7 & 8 & 9 & 10 \\
\hline \multirow{10}{*}{$\begin{array}{l}\stackrel{0}{0} \\
\overrightarrow{0} \\
\overrightarrow{0}\end{array}$} & 1 & 37 & 18 & 3 & 2 & 0 & 0 & 0 & 0 & 0 & 0 \\
\hline & 2 & 19 & 23 & 10 & 8 & 1 & 1 & 0 & 0 & 0 & 0 \\
\hline & 3 & 4 & 15 & 20 & 17 & 5 & 0 & 0 & 0 & 0 & 0 \\
\hline & 4 & 0 & 4 & 16 & 16 & 14 & 8 & 1 & 1 & 1 & 0 \\
\hline & 5 & 0 & 1 & 10 & 9 & 20 & 11 & 8 & 2 & 0 & 0 \\
\hline & 6 & 0 & 0 & 0 & 6 & 14 & 24 & 12 & 5 & 0 & 0 \\
\hline & 7 & 0 & 1 & 1 & 3 & 4 & 13 & 18 & 19 & 2 & 0 \\
\hline & 8 & 0 & 0 & 1 & 0 & 0 & 3 & 16 & 20 & 16 & 5 \\
\hline & 9 & 0 & 0 & 0 & 0 & 3 & 1 & 4 & 11 & 23 & 19 \\
\hline & 10 & 0 & 0 & 0 & 0 & 0 & 0 & 2 & 3 & 19 & 38 \\
\hline
\end{tabular}

GMDND = ganho médio diário na pré-desmama; PD160 = precocidade em dias para ganhar $160 \mathrm{~kg}$.

Tabela 4 - Mudança de classificação em decas com o uso dos critérios de seleção GMDND e PD160c para 611 touros da raça Guzerá

\begin{tabular}{|c|c|c|c|c|c|c|c|c|c|c|c|}
\hline \multirow[t]{2}{*}{ Seleção } & \multicolumn{11}{|c|}{ GMDND } \\
\hline & Decas & 1 & 2 & 3 & 4 & 5 & 6 & 7 & 8 & 9 & 10 \\
\hline \multirow{9}{*}{$\begin{array}{l}0 \\
0 \\
0 \\
\hat{0} \\
\hat{a}\end{array}$} & 1 & 48 & 12 & 0 & 0 & 0 & 0 & 0 & 0 & 0 & 0 \\
\hline & 3 & 2 & 8 & 26 & 21 & 4 & 0 & 0 & 0 & 0 & 0 \\
\hline & 4 & 0 & 8 & 11 & 19 & 17 & 6 & 0 & 0 & 0 & 0 \\
\hline & 5 & 0 & 0 & 5 & 8 & 24 & 19 & 5 & 0 & 0 & 0 \\
\hline & 6 & 0 & 1 & 2 & 5 & 7 & 25 & 21 & 0 & 0 & 0 \\
\hline & 7 & 0 & 0 & 1 & 2 & 5 & 5 & 25 & 22 & 1 & 0 \\
\hline & 8 & 0 & 0 & 1 & 2 & 3 & 4 & 6 & 44 & 18 & 0 \\
\hline & 9 & 0 & 0 & 1 & 0 & 0 & 2 & 3 & 10 & 34 & 11 \\
\hline & 10 & 0 & 0 & 0 & 0 & 0 & 0 & 1 & 2 & 8 & 51 \\
\hline
\end{tabular}

GMDND = ganho médio diário na pré-desmama; PD160 = precocidade em dias para ganhar 160 kg; PD160c = PD160 corrigido para o efeito de grupo de contemporâneos. 
Tabela 5 - Resumo da análise de variância do desvio-padrão ( $\sigma)$ do GMDNDc de bovinos da raça Guzerá

\begin{tabular}{lcc}
\hline Fonte de variação & GL & Quadrado médio \\
\hline$\sigma$ (linear) & 1 & $0,00018^{\text {ns }}$ \\
$\sigma$ (quadrático) & 1 & $0,04152^{* * *}$ \\
Resíduo & 609 & 0,00007 \\
Total & 611 & \\
\hline
\end{tabular}

GMDNDc = ganho médio diário na pré-desmama corrigido para o efeito de grupo de contemporâneos.

$\left({ }^{\text {ns }) ~ N a ̃ o-s i g n i f i c a t i v o ~ a ~} 0,01 ;(*)\right.$ Significativo $\mathrm{P}<0,001$.

\section{Conclusões}

Ganho médio diário na pré-desmama e precocidade em dias para ganhar 160 kg apresentaram alta correlação genética, indicando que ambas as características respondem à seleção para a precocidade de crescimento. A seleção para precocidade em dias para ganhar $160 \mathrm{~kg}$ favorece touros que produzem progênie com desempenho superior e menos

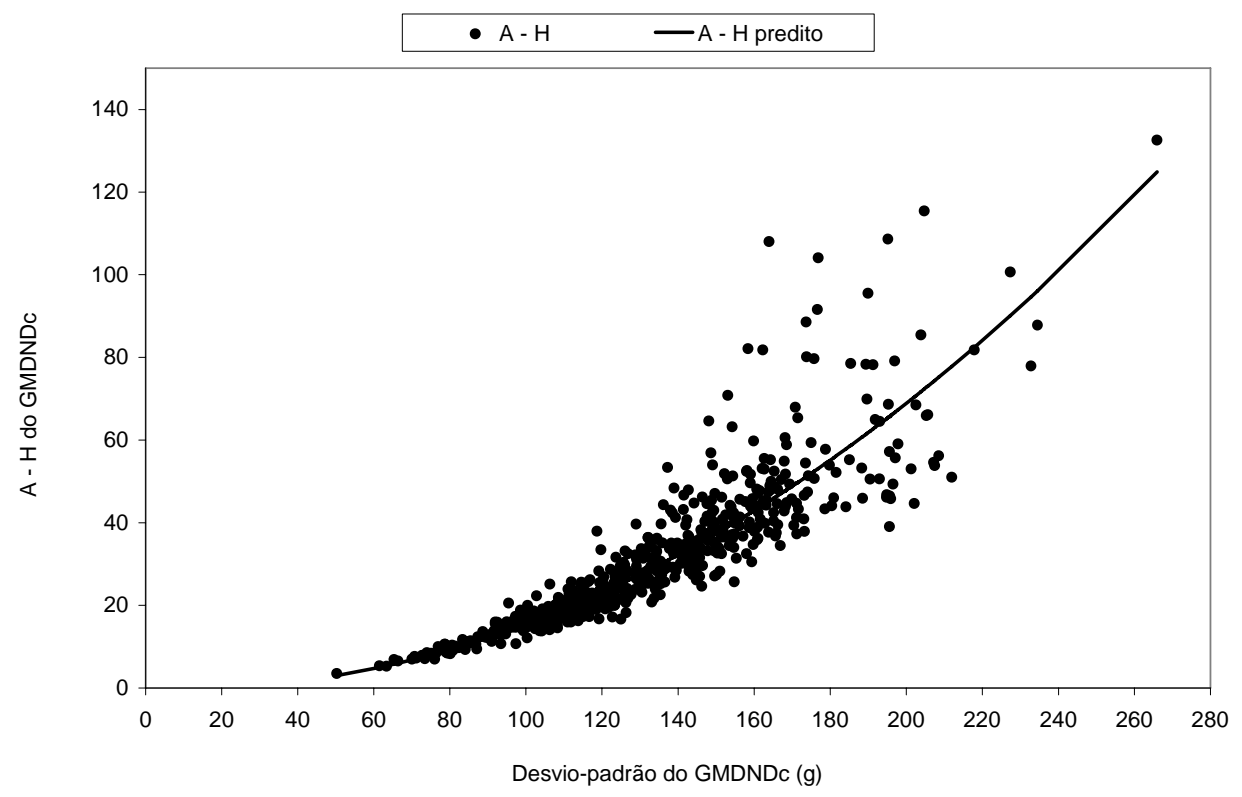

Figura 3 - Diferença entre a média aritmética e média harmônica (A-H) e desvio-padrão ( $\sigma$ ) do ganho médio diário na pré-desmama corrigido para o efeito de grupo de contemporâneos (GMDNDc), em g/dia, de animais da raça Guzerá.

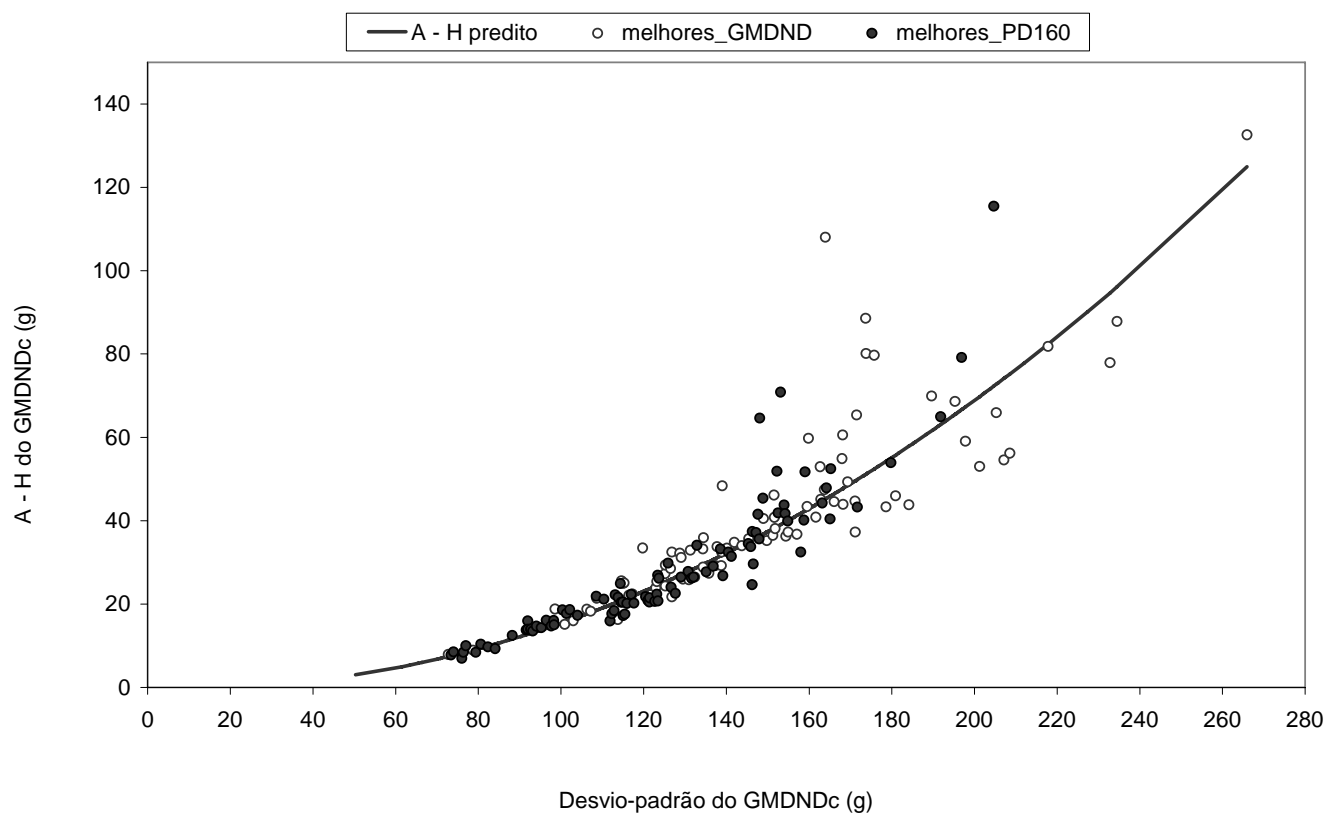

Figura 4 - Diferença entre as médias aritmética e harmônica (A-H) e desvio-padrão do ganho médio diário na pré-desmama corrigido para o efeito de grupo de contemporâneos (GMDNDc) de touros mais bem classificados para GMDNDc e para precocidade em dias para ganhar $160 \mathrm{~kg}$ (PD160). 


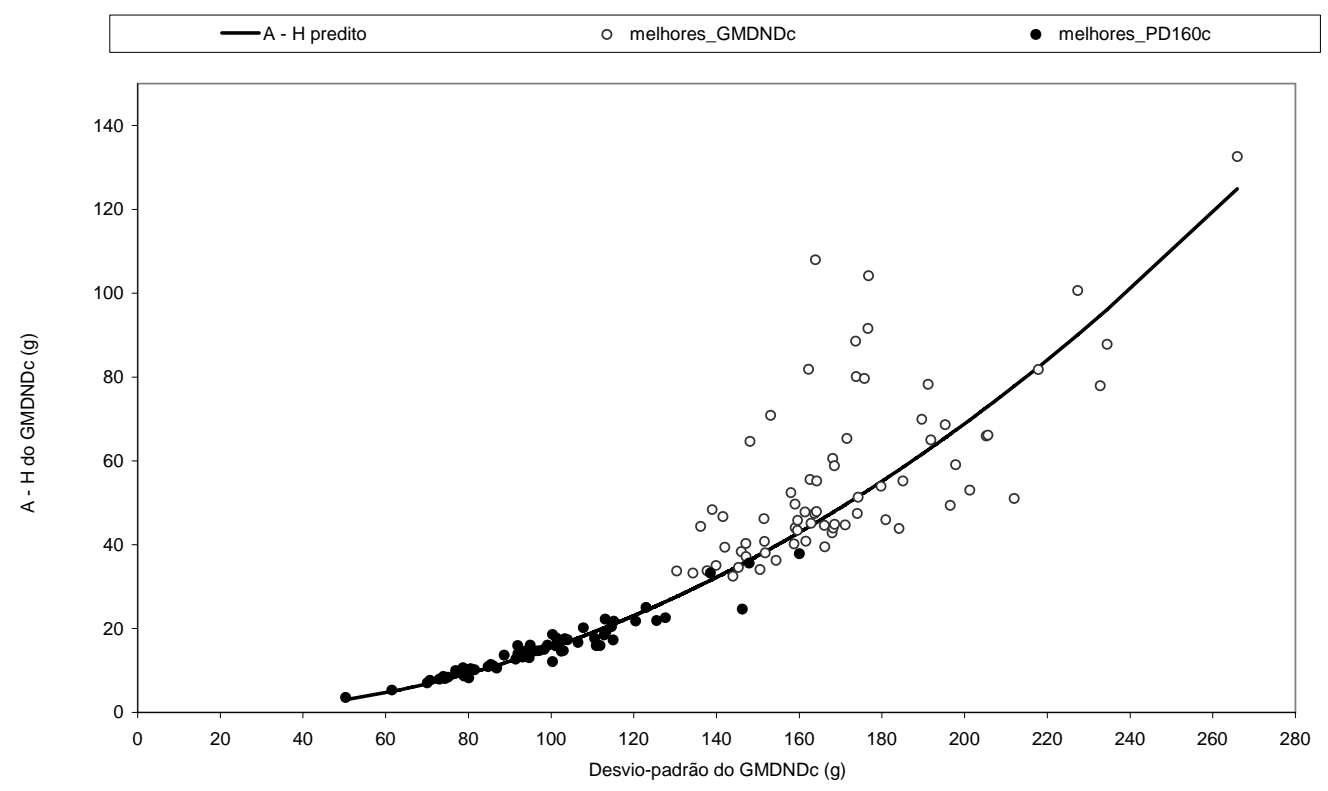

Figura 5 - Diferença entre as médias aritmética e harmônica (A-H) e desvio-padrão do ganho médio diário na pré-desmama, corrigido para o efeito de grupo de contemporâneos (GMDNDc) dos touros mais bem classificados para GMDNDc e para PD160 corrigido para o efeito de grupo de contemporâneos (PD160c).

variável e a padronização da característica com base no grupo de contemporâneos melhorou sua eficiência. A classificação dos touros variou de acordo com o critério de seleção utilizado, ganho médio diário de peso ou precocidade em dias para ganhar $160 \mathrm{~kg}$, principalmente nos extremos, ou seja, nas faixas onde ocorrem a seleção e descarte de reprodutores.

\section{Agradecimentos}

À Associação Brasileira de Criadores de Zebu, pela concessão dos dados utilizados neste estudo, e ao CNPq, pela bolsa de iniciação científica da primeira autora.

\section{Literatura Citada}

BOLDMAN, K.G.; KRIESE, L.A.; Van VLECK, L.D. A manual for use MTDFREML. Nebrasca: Clay Center; USDA-ARS, 1993. 120p.

CARVALHEIRO, R.; ORTIZ PEÑA, C.D.; QUEIROZ, S.A. et al. Alternativa de avaliação genética para critérios de seleção baseados em funções da média harmônica. In: REUNIÃO ANUAL DA SOCIEDADE BRASILEIRA DE ZOOTECNIA, 40., 2003, Santa Maria. Anais... Santa Maria: Sociedade Brasileira de Zootecnia, 2003. (CD-ROM).

FRIES, L.A.; BRITO, F.V.; ALBUQUERQUE, L.G. Possíveis conseqüências na seleção para incrementar pesos às idadespadrão vs. reduzir idades para produzir unidades de mercado. In: REUNIÃO ANUAL DA SOCIEDADE BRASILEIRA DE ZOOTECNIA, 30., 1996, Fortaleza. Anais... Fortaleza: Sociedade Brasileira de Zootecnia, 1996. p.310-312.
FRIES, L.A.; ALBUQUERQUE, L.G. Avaliação genética de duas alternativas para medir precocidade de crescimento. In: SIMPÓSIO NACIONAL DE MELHORAMENTO ANIMAL, 1., 1996, Ribeirão Preto. Anais... Ribeirão Preto: Sociedade Brasileira de Melhoramento Animal, 1996. p.243-245.

HENDERSON, C.R. Applications of linear models in animal breeding. Ontario: University of Guelph, 1984. 462p.

MUNIZ, C.A.S.D.; CARVALHEIRO, R.; FRIES, L.A. et al. Dois critérios de seleção na pré-desmama de bovinos da raça Gir. 1. Estimativas de parâmetros genéticos. Revista Brasileira de Zootecnia, v.34, n.3, p.807-815, 2005a.

MUNIZ, C.A.S.D.; CARVALHEIRO, R.; FRIES, L.A. et al. Dois critérios de seleção na pré-desmama de bovinos da raça Gir. 2. Efeito na classificação dos animais. Revista Brasileira de Zootecnia, v.34, n.3, p.816-826, 2005b.

ORTIZ PEÑA, C.D.; QUEIROZ, S.A.; FRIES, L. Comparação entre critérios de seleção de precocidade sexual e a associação destes com características de crescimento em bovinos Nelore. Revista Brasileira de Zootecnia, v.30, n.1, p.93-100, 2001.

ORTIZ PEÑA, C.D.; CARVALHEIRO, R.; QUEIROZ, S.A. et al. Comparison of selection criteria for pré-weaning growth traits of Nelore cattle. Livestock Production Science, v.86, p.163-167, 2004.

PITA, F.V.C.; ALBUQUERQUE, L.G. Resposta à seleção para características de desempenho em um rebanho de seleção de suínos. Revista Brasileira de Zootecnia, v.30, n.6, p.20092016, 2001.

ROSO, V.M. Alternativas para avaliar taxas de crescimento e conseqüências na seleção de suínos. In: REUNIÃO ANUAL DA SOCIEDADE BRASILEIRA DE ZOOTECNIA, 34., 1997, Juiz de Fora. Anais... Juiz de Fora: Sociedade Brasileira de Zootecnia, 1997. p.308-310.

STATISTIC ANALYSIS SYSTEM - SAS. SAS/STAT software. User's Guide. version 6.11, 4.ed., v.2, Cary: SAS Institute, 1996. 842p.

SCHAEFFER, L.R. Quantitative genetic and animal models: Repeated records animal models. Ontario: University of Guelph, 2003. p.1-11. (Course notes). 